MEINEM LIEBEN SCHWAGER,

DEM RITTERGUTSBESITZER

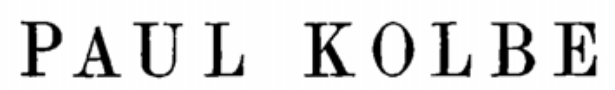

AUF BLESEWITZ IN POMMERN,

UNTER DESSEN GASTIICHEM DACHE SO MANCHE AUFLAGE DIESES BUCHES ZUSTANDE GEKOMMEN IST,

IN DANKBARER LIEBE UND FREUNDSCHAFT

ZUGEEIGNET. 



\title{
LEHRBUCH
}

DER

\section{DEUTSCHEN RECHTSGESCHICHTE}

VON

\author{
DR. RICHARD SCHRÖDER \\ PROFESSOR IN HEIDELBERG
}

FUNFTE, VERBESSERTE AUFLAGE

MIT EINER ABBILDUNG IM TEXT UND FÜNF KARTEN

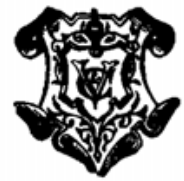

LEIPZIG

VERLAG VON VEIT \& COMP. 
Druck von Metzger \& Wittig in Leipzig. 